\title{
The Design and Implementation of Mobile Online Testing System based on Android Platform
}

\author{
Xiangyi Li \\ Information technology department \\ QiongTai Teachers College \\ Haikou,China \\ 58628511@qq.com
}

\author{
Yanheng Li \\ Information Science Technology Department \\ Dalian Maritime University \\ Dalian Liaoning, China \\ 28046643307@qq.com
}

\begin{abstract}
The development of the system is mainly for students in the Android mobile platform for online testing of each course.This paper first gives the environment of the development of the testing system, analyzes the system structure of the online testing system, expounds the basic development ideas of the system, focuses on the entity class, layout file, activity class, data layer and business layer.The relationship of the data layer, activity, service layer three is analyzed. The implementation steps of the system are listed, and the three key algorithms used in the development process are described, which are parsing the test questions, scrambling the order of questions, and showing the remaining time algorithm.
\end{abstract}

Keywords-System;Android;Algorithm;Class;Entity;

\section{INTRODUCTION}

Android is a free and open source operating system based on Linux, which is developed by Google and the open mobile phone alliance. Android's opening is no doubt to the developers, so that developers can understand the internal structure of the Android, better development, and can be based on the project needs to make appropriate changes[2].

But due to the Android platform provides the component development pattern for developers,the difficulty of the project application developers to develop not only did not increase but also decrease, plus the use of eclipse Java integrated development environment (IDE), let the Android platform become now mobile device of choice.

In recent years, mobile learning has been widely used in educational training of schools, enterprises and other organizations. As the increasing of the mobile devices, the advantage of the full use of the learners attracts more and more social attention, which can improve the learning efficiency and strengthen the classroom teaching objectives[3].

In the school, the exam is not only a means to check the students learning situation, but also an important learning link, plays the role of baton. With the development of communication technology and mobile technology, smart phones in the school is becoming more and more popular, Android equipment has the advantage of price, while its performance is not lost, with a very high cost performance. In order to improve the existing exam mode, to create a new test environment for students, and to track the students' learning situation in time, the online testing system based on Android is developed[8].At the same time, compared with the traditional online exam, in the exam of the convenience and cost control, the use of Android equipment as a test client has a considerable advantage.

The online testing system is convenient, fast, and can readily be used to carry out the test of knowledge points. Comparing with the traditional written exam, it saves human and material resources; Comparing with the test on the computer ,it saves time, breaks the space, in the classroom, dormitory, library and other places can be tested.

\section{CONFIGURATION OF THE ONLINE TESTING SYSTEM}

Before the development of the exam system, the first to build a Android development environment.

Download JDK (java development environment package), configure environment variable;

JDK that is the core of the whole Java Development Kit Java, including a series of Java development. After the installation, you need to configure the environment variable.

The variable JAVA_HOME itself does not exist, and needs to be created. \%JAVA_HOME can be used as a unified reference path, the value of the JDk installation directory;

The variable PATH already exists, you can edit directly. Add the value of a variable in PATH. Its value is \%JAVA_HOME\%lbin;\%JAVA_HOME\%ljrelbin;

The variable CLASSPATH itself does not exist, and needs to be created. JAVA class path for compilation. The variable is set to two values, which is the JVM search for the current directory.The value is.;\%JAVA_HOME5 \lib\tools.jar.

The installation of Eclipse; Eclipse is the IDE of Java and Android that developed.

The installation of SDK (software development kit), SDK is the Android management development kit tool, provides the development kit and tools for the Android platform;

The installation configuration of ADT; 
Create an AVD view.

After the above five steps, the Android environment has been built, next begin to develop the online testing system.

\section{ARCHITECTURE OF THE ONLINE TESTING S YSTEM}

The online testing system is divided into server and client, the server uses HFS, the questions and the user is stored in the form of text file, and the exam questions and user information are uploaded to the server. The client can carry out the corresponding on-line evaluation The functional structure of the system is shown in Fig .1.

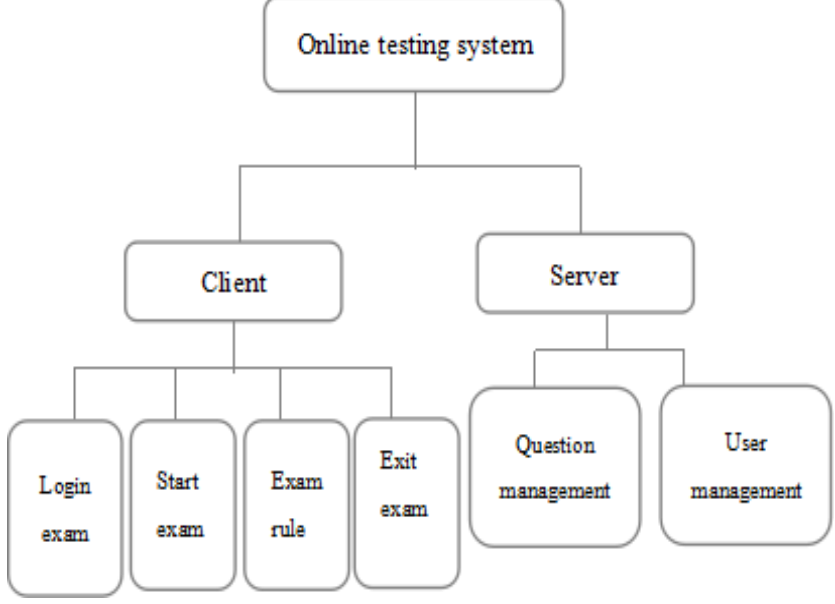

Figure 1. Online testing system structure diagram

\section{BASIC DEVELOPMENT IDEA OF ONLINE TESTING SYSTEM}

\section{A. First determine the entity class;}

Through the analysis of the structure of the online testing system, it can be seen that the system mainly has three major entities, the user entity, the exam entity, the exam information entity. So you need to create a folder to store the three entity, and establish the corresponding entity class, which named User.java, Question.java, ExamInfo.java.

\section{B. Then determine the layout file;}

The system mainly contains user login, the main interface and the beginning of the exam,so it is necessary to build three layout file. File names were Login.xml, Main.xml, Exam.xml.

\section{Determine the activity file;}

In the Android application, the page basic unit is Activity, which is the presentation layer of the page control, the user to display the visual user interface, and the corresponding interface, similar to the from in the web or desktop editing, usually for a separate screen. This system mainly has three activity, File name is LoginActivity.java, MainActivity.java, ExamActivity.java .

\section{The establish of data layer and service layer}

In order to interact the server directly,we need to create the data layer,the new interface in the data layer and the realization of the interface class. To define the method of defining query user information and loading method in the interface, and the method of loading test information ,then implement these methods in specific class.

And to interact the activity directly, we need to create a service layer, in the service layer to create interface and interface to achieve the class and exception. The service layer handles the service logic of the activity, while the resource is called in the data layer.

Interaction flow diagram between them is shown in Fig. 2 .

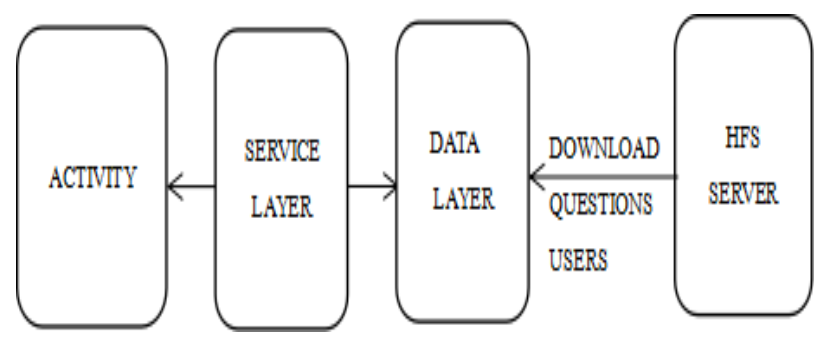

Figure 2. interaction flow diagram

\section{DEVELOPMENT STEPS OF THE ONLINE TESTING SYSTEM}

\section{A. Start server}

First of all,we need to create a new Android project named exam in eclipse, and a new com.qttc.exam package in the src folder.Copy all the picture materials which are used in the online testing system to the "drawable" directory. Store the user information and the exam information in a text file, before the exam, we need to start the server (HFS), loading the user information and exam information.

\section{B. Create layout page}

Set up the layout page in the "exam/res/layout " folder, land the layout page login.xml, the main interface main_menu.xml, start the exam interface exam.xml. In the establishment of the layout of the page, the layout style is the flow layout, set the ID name of the control , the width and height of the control respectively.

\section{Create entity class}

Create folder called entity in "exam/src" , create three entity classes in the folder,first, the user entity User.java, the class is a JavaBean object, to create a private variable: ID, name, password, phone number, email, create set method and get method. Then exam entity.Question.java, respectively, to create private variables ID, questions of title, options, a fraction, the difficulty level, correct answer, the answer,selected by user, Among them, store correct answers and user choice answers with linear container. Finally,set up exam information creating entity class ExamInfo.java, create test category, user name, exam time and exam number.

\section{Create data layer}

Create a folder named data in "exam/src"; Create an interface class IExamData.java in the folder, define constants in the interface, and these constants are the file names and 
addresses downloaded from the HFS server, define some abstract methods, including the search for user information, loading the database, access to all questions, load test information, and access to the test information.

Create "ExamData.java" in the folder to implement the interface IExamData, in which define the user object, Create HashMap objects in the non parametric method, assign for users, then download the user.txt file from HFS server, and look up all the user information in the file,finally put into the user container. Also create the questions container object, download the java.txt from the server, read the contents of the file,parse as Question object, and load the questions . How to parse question is the key part, but also the difficulty, in the back of the key algorithm to give a specific description.

\section{E. Create service layer}

Create service folder in "exam/src", in the folder create the interface IExamService.java.The function of the interface : a method to judge the login authentication, get the user object, the purpose is in order to complete the function that welcome user to take the exam; loading questions and get questions, start test and scramble the order of the questions and display one question and save the answer chose by user, judging and scoring.

In the folder create "ExamService.java" implement interfaces IExamService, implement the abstract method of the interface, The algorithms of scramble the order of the questions will be described in the behind of the key algorithms.

\section{F. $\quad$ Create activity}

Create a folder activity in "exam/src", create a new "LoginActivity.java" in the folder, in the initialization method of the class, get the control ID at the login interface, and create a thread, creating a ExamService in the thread's run method. Login button and exit button to click on the event monitor.

Create "MainMenuActivity.java" in the folder, in the initialization method of the class, assign examService of this class with examService that transmitted from LoginActivity through the intent class and bundle class, get the ID of starting exam button, and bind the exam button on event listener. In the method of setListener, when you start the exam, you need to download the questions, use the thread to complete this function. The run method of the thread, load questions through the service layer,the rest of the work will be completed by the next Activity ,transmit examService to ExamActivity through the bundle class.

Create "ExamActivity.java" in the folder,define the TextViews of exam information, display questions and exam information, and remaining time in the class,define a array used to store the four check boxes;

Get these controls in the initialize method by Id; Get the examService transmitted from MainMenuActivity and get all questions from the thread, and start the exam; get the exam time that specified in the exam information and carry out exam countdown; bind last question, next question, and submit button on event listener.

The interaction among the activity is shown in Fig.3.

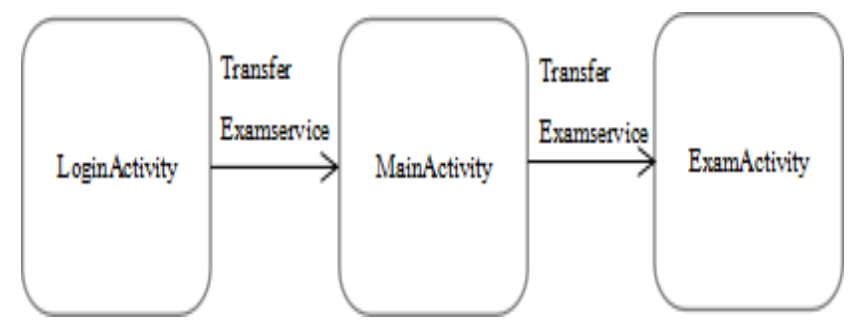

Figure 3. Activity interaction diagram

\section{G. Configure AndroidManifest.xml project list}

Configure the three activity from the sixth step to the project list, and set the LoginActivity as main activity.

\section{KEY ALGORITHM OF THE ONLINE TESTING SYSTEM}

The development of online testing system involves some key algorithms,download the questions from the server.when design start exam interface, how to parse the questions from java.txt, and scramble the order of questions while loading questions, showing the time of the test while starting exam .The start exam interface is shown in Fig .4.

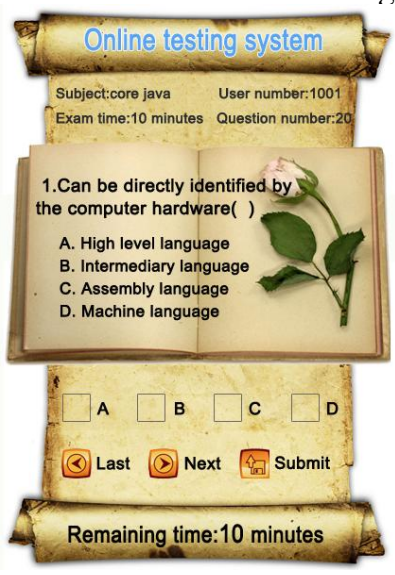

Figure 4.The start exam interface

Three key algorithms are described below.

Algorithm description of parsing questions; public Question parseQuestion(BufferedReader reader, String line) throws IOException \{

Question q = new Question();

String[] data = line.split(",");

ArrayList $<$ String $>$ answers = new ArrayList $<$ String $>()$; String[] strsAns = data[0].split(":")[1].split("/");

for (int $\mathrm{i}=0 ; \mathrm{i}<$ strsAns.length; $\mathrm{i}++)\{$

answers.add(strsAns[i]); \}

q.setAnswers(answers);

q.setScore(Integer.parseInt(data[1].split(":")[1]));

q.setLevel(Integer.parseInt(data[2].split(":")[1]));

//parse the first line,user reader parse the next line.

q.setTitle(reader.readLine());

// read four options

String options $=$ "";

for (int $\mathrm{i}=0 ; \mathrm{i}<4 ; \mathrm{i}++)$ \{

options += reader.readLine ()$+$ "\n"; 
\}

q.setOptions(options); return q;

\}

2)Algorithm description of scrambling the order of questions;

public ExamInfo startExam() \{

//scramble the order of the questions randomly

Collections.shuffle(questions, new Random());

// regenerate the tiltle of the question

for (int $\mathrm{i}=0$; $\mathrm{i}<$ questions.size ()$; \mathrm{i}++)\{$

Question question = questions.get(i);

String title = question.getTitle();

int index = title.indexOf(".");

// Cover the original title

title $=(i+1)+$ title.substring $($ index $)$;

question.setTitle(title); $\}$

examInfo = examDao.findExamInfo(user.getId());

return examInfo;

Algorithm description of displaying the remaining time of exam.

public void startTime ()\{

// obtain the exam time

long limitTime = examInfo.getLimitTime ()$* 60 * 1000$;

// convert to milliseconds

final long end $=$ limitTime + System. currentTimeMillis();

// finish time milliseconds

final Timer timer $=$ new Timer();

timer.schedule(new TimerTask() \{

long minute;

long second;

public void run() \{

long time = end - System.currentTimeMillis();

minute $=$ time $/ 1000 / 60 ; / /$ minute

second $=$ time $/ 1000 \% 60 ; / /$ second

\}$, 0,1000)$;

\section{CONCLUSION}

The introduction of the evaluation system is aimed at entering the field of mobile education,providing a better choice for student' mobile phones and other mobile terminals . Students can make full use of spare time,exercises whenever and wherever possible. This system reflects the guiding ideology of the students as the teaching center, the "learning and doing",promote students' learning enthusiasm through "the practice of teaching", optimize teaching efficiency. The next research focus will be on data analysis and data mining, so that teachers can easily see the interest of students learning, as well as the problems encountered in the process of learning. However, the function of the system is relatively simple, and the function of the system is to be expanded and improved in the following work.

\section{REFERENCES}

[1] Zhu Shuai. The research of natinal computer grade exam system based on Android [J]. Electronic world, 2014,PP.407-408.

[2] Wu Yuanjun. Development and implementation of mobile learning platform based on Android[J]. Journal of Chuzhou University, 2015 (2),PP. 59-62.

[3] Wu Kaicheng, Zhang Si, Liao Enyang. Design and implementation of online exam system based on Android[J]. Computer age, 2015 (3),PP.27-29.

[4] Ma Xueshan.Design and impementation of CCT mobile learing system based on Android platform $[\mathrm{J}]$. Electronic technology and software engineering, 2015,PP.75-76.

[5] Han Lingbo. Design and implementation of a cadre mobile learning system based on Android platform [J]. modern computer, 2014 (6),PP. 72-74.

[6] Huang Junshi. Best practice of Android and PHP development [M]. Beijing: Mechanical Industry Press, 2013.

[7] Qin Wenwen.Computer theory exam collection based on Android platform[J].Computer programming skills and maintenance.2014(09),pp.28-29.

[8] Wu Baixiong. The application of mobile phone test system based on Android platform [J].Application of computer system,2014 (12),pp. 72-76.

[9] Zhang Chengjie. The design and implementation of online testing system based on Android platform[J].Computer applications and software, 2014(4) ,pp.323-325.

[10] Gao Aimei.Design and implementation of an online exam system based on Android[J].Information education,2015 (9),pp. 107-108. 